\title{
Voluntary exercise in mesothelioma: effects on tumour growth and treatment response in a murine model
}

Scott A. Fisher ${ }^{1,2^{*}+}$ (D), Carolyn J. Peddle-McIntyre ${ }^{3,4+}$, Kimberley Burton ${ }^{1,2}$, Robert U. Newton $3,4,5$, Elly Marcq ${ }^{6}$, Richard A. Lake ${ }^{1}$ and Anna K. Nowak ${ }^{1,7}$

\begin{abstract}
Objective: There is substantial evidence that exercise can safely reduce the risk of cancer and improve survival in different human cancer populations. Long latency periods associated with carcinogen-induced cancers like asbestos induced mesothelioma provide an opportunity to implement exercise as an intervention to delay or prevent disease development. However, there are limited studies investigating the ability of exercise to prevent or delay cancer, and exercise as a preventive strategy has never been assessed in models with a known carcinogen. We investigated the potential of voluntary exercise (VE) to delay development of asbestos related disease (ARD) in our well-characterised, asbestos induced MexTAg model of mesothelioma.

Results: Asbestos exposed MexTAg mice were given continuous or delayed access to VE and ARD assessed over time. We found that the addition of VE did not affect ARD development in asbestos exposed MexTAg mice. However, non-asbestos exposed, aged matched control mice participated in significantly more VE behaviours, suggesting subclinical development of ARD after asbestos exposure had a greater impact on VE participation than age alone. These data highlight the importance of model choice and the potential limitation that some pre-clinical studies may not accurately represent the clinical paradigm, particularly in the context of prevention studies.
\end{abstract}

Keywords: Asbestos, Mesothelioma, Exercise, MexTAg

\section{Introduction}

Mesothelioma is an asbestos induced cancer with poor prognosis. Treatment is usually palliative, with systemic therapy providing limited survival benefit $[1,2]$. Despite mesothelioma having a long latent period (20-40 years) between asbestos exposure and diagnosis [3], there are currently no effective strategies to prevent mesothelioma onset after asbestos exposure. Carcinogen induced cancers progress through multiple genetic, epigenetic and

\footnotetext{
*Correspondence: scott.fisher@uwa.edu.au

${ }^{\dagger}$ Scott A. Fisher, Carolyn J. Peddle-Mclntyre contributed equally to this work

${ }^{2}$ School of Biomedical Sciences, University of Western Australia, Perth, Australia

Full list of author information is available at the end of the article
}

immunological modification, culminating in clinically apparent disease [4]. It is logical to hypothesise that some intervention during disease latency might delay onset of, or even prevent mesothelioma development. Such studies in humans require large participant numbers and take many years. However, animal studies allow for pre-clinical testing of these hypotheses, and subsequent selection of effective treatments for clinical trials.

Strong epidemiological evidence indicates that physical activity is associated with a reduced risk of developing cancer [5]. Compared with being inactive, the highest levels of physical activity have been associated with a $25 \%$ reduction in incidence of breast and colorectal cancer $[6$, 7]. Collectively, epidemiological evidence suggests that physical activity has tumour-mitigating properties across

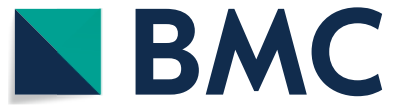

(c) The Author(s) 2020. This article is licensed under a Creative Commons Attribution 4.0 International License, which permits use, sharing, adaptation, distribution and reproduction in any medium or format, as long as you give appropriate credit to the original author(s) and the source, provide a link to the Creative Commons licence, and indicate if changes were made. The images or other third party material in this article are included in the article's Creative Commons licence, unless indicated otherwise in a credit line to the material. If material is not included in the article's Creative Commons licence and your intended use is not permitted by statutory regulation or exceeds the permitted use, you will need to obtain permission directly from the copyright holder. To view a copy of this licence, visit http://creativeco mmons.org/licenses/by/4.0/. The Creative Commons Public Domain Dedication waiver (http://creativecommons.org/publicdomain/ zero/1.0/) applies to the data made available in this article, unless otherwise stated in a credit line to the data. 
many cancers. Exercise holds potential as an intervention to delay or prevent mesothelioma in asbestos exposed individuals. However, the ability of exercise to prevent or delay cancer has never been investigated in models with a known carcinogen.

Pre-clinical evidence demonstrates that voluntary exercise (VE) can suppress tumour initiation and growth in mouse models of breast, colorectal and lung cancer [8-13]. Exercise induced suppression of tumour initiation and growth may be mediated by effects on tumour metabolism and immune function. Inflammation is critical in cancer development and VE has been shown to attenuate the inflammatory response in mice following carcinogen exposure, promoting more efficient clearance of damaged cells [14]. Additionally, VE decreases tumour incidence and growth via regulation of key immune cells, including natural killer (NK) and effector lymphocytes [13]. Here we investigated the potential of VE to delay development of asbestos related disease (ARD) in our well-characterised, asbestos induced MexTAg mouse cancer model $[15,16]$.

\section{Main text \\ Methods \\ Experimental design}

Experiments were approved by the UWA AEC (RA/3/100/1514) in accordance with the Australian code for the use of animals in medical research [17]. The C57Bl/6 derived 266 MexTAg transgenic mouse model expressing the Simian Virus 40 (SV40) Large T antigen (TAg) under the control of the mesothelin promoter has been described [15, 16]. For all experiments MexTAg mice (28-30/group, M\&F, 6-20 weeks) were randomly assigned to their experimental groups and housed 3 per cage (Thoren cage; $19.56 \times 30.91 \times 13.34 \mathrm{~cm}$ ) under standard conditions with food (standard meat free mouse and rat diet. Specialty feeds, Perth W.A. Cereal grain based diet, $12 \mathrm{~mm}$ pellets, digestible energy $14 \mathrm{MJ} / \mathrm{kg}$ ) and water provided ad libitum. Mice were euthanised via methoxyflurane anaesthesia immediately prior to cervical dislocation. The extended duration of experiments and overt nature of ARD prevented blinding of investigators to treatment groups.

\section{MexTAg mice and asbestos instillation}

MexTAg mice were exposed to crocidolite asbestos via two, $0.5 \mathrm{ml}(3 \mathrm{mg})$ intraperitoneal (i.p.) injections per mouse at weeks 0 and 4, monitored at least twice weekly and euthanised when ARD became evident; commonly ascites induced abdominal distension. ARD development and mesothelioma incidence was confirmed on histology as previously described $[15,16]$.

\section{Voluntary exercise (VE)}

VE was assessed by placing an activated or locked low profile wireless running wheel (Med Associates Inc ${ }^{\circledR}$, USA.) into each cage and data collected using the USB Interface Hub and computer running SOF 860 Wheel Manager software. Mice were acclimatised and trained on running wheels for 1 week prior to start of experiment. Actogram analysis was performed using SOF 861 Wheel Analysis software, while assessment of VE parameters such as time spent running and distance run was performed using Microsoft Excel. Data was collected $24 / 7$.

\section{Statistics}

Comparisons between two individual, or three or more groups were performed using unpaired, nonparametric (Mann-Whitney) Student's $t$ test (with Holm-Sidak correction for multiple comparisons), or unpaired, ordinary one-way ANOVA with Dunnett's correction for multiple comparison respectively, relative to first data point. Log rank (Mantel-Cox) analysis was performed on survival curves. Analyses were performed using Graph Pad Prism Software V8 (Graph Pad Software Inc., USA). We used R [18] with afex [19] and emmeans [20] packages to perform mixed model ANOVA to analyse differences in VE behaviour over time. $\mathrm{p}$ values $\leq 0.05$ were considered significant.

\section{Results}

\section{Nocturnal peak in voluntary exercise}

To assess the impact of voluntary exercise on ARD, asbestos exposed MexTAg mice (28-30/group) were randomly assigned to No VE or VE groups that either had continuous access to activated running wheels (Pre-exposure group (Pre-VE); starting 2 weeks before asbestos instillation), or from 25 weeks after asbestos instillation (Post exposure group (Post-VE), Fig. 1a). Age matched, non-asbestos exposed MexTAg mice served as controls to assess changes in exercise behaviour over time in the absence of asbestos (Fig. 1b). All mice were assessed for overall survival, while asbestos exposed mice were additionally assessed for disease latency; time from asbestos exposure to first signs of disease (FSD) and disease progression; time from FSD until cull.

Although voluntary exercise activity was collected continuously throughout the experiment, we first sought to identify the circadian pattern of VE. We observed high levels of running wheel activity between $2200 \mathrm{~h}$ and $0600 \mathrm{~h}$, within any $24 \mathrm{~h}$ period for all VE groups (Fig. 1c). Based on these findings, all subsequent analysis of VE 


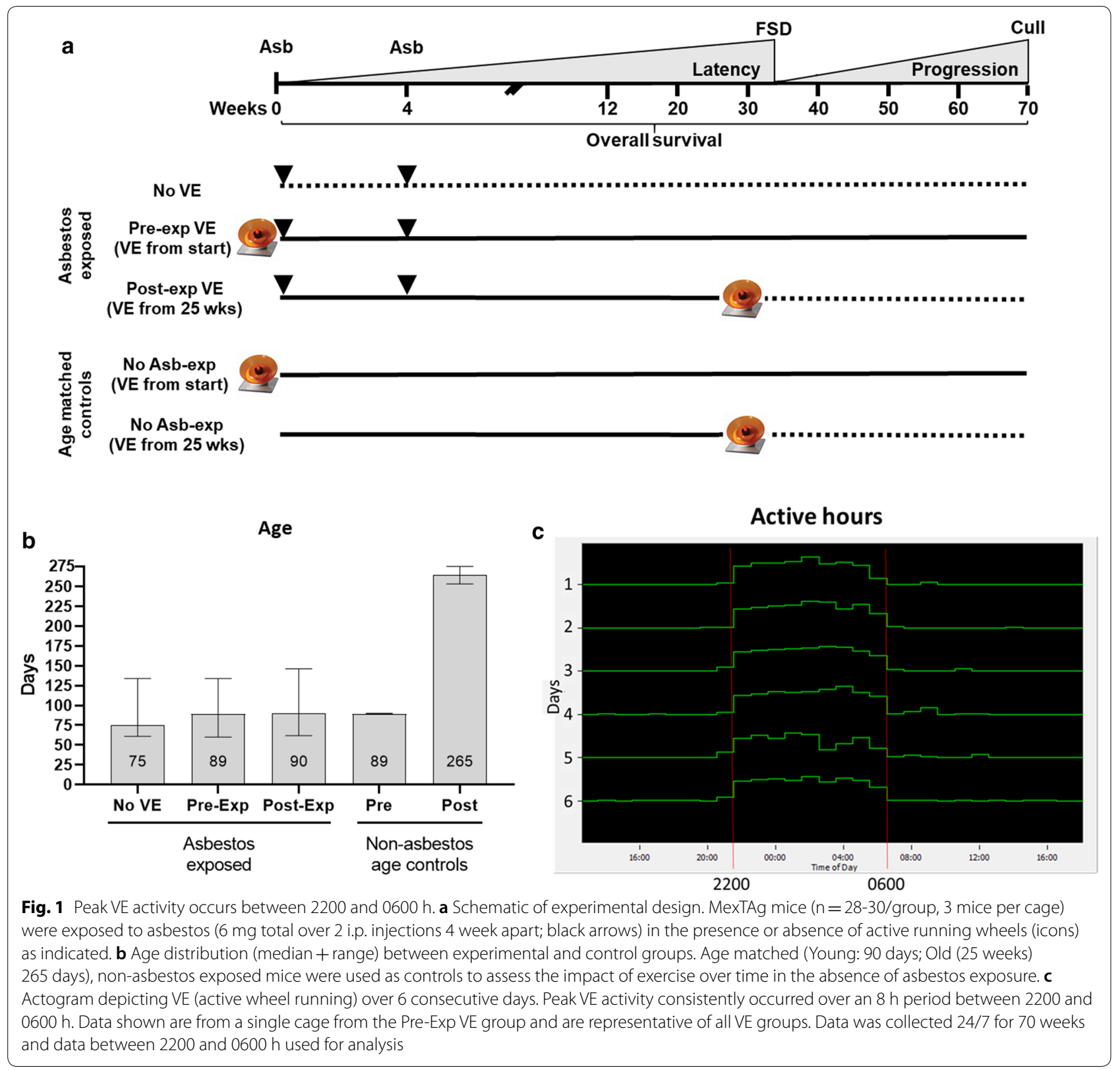

parameters was performed on data obtained within this period.

\section{Voluntary exercise does not affect asbestos related disease development in MexTAg mice}

No difference in median survival was observed between asbestos exposed mice given access to VE compared to the No VE control group (Pre-VE vs. No VE; 39.9 vs. 45.3 weeks, $\mathrm{p}=0.2784$; Post-VE vs. No VE; 44.9 vs. 45.3 weeks $\mathrm{p}=0.7404$ ), with all but 3 mice ( 2 from No $\mathrm{VE}$ and 1 from Post VE) succumbing to ARD by 70 weeks (Fig. 2a). No difference was observed in disease latency between asbestos exposed groups relative to No VE controls (Pre-VE 39.3 weeks $(\mathrm{p}=0.72)$; Post-VE 42.9 weeks $(\mathrm{p}<0.99$ vs. No VE 43.1 weeks), or disease progression (Pre-VE 0.3 weeks $(\mathrm{p}<0.99)$, Post-VE 0.1 weeks $(\mathrm{p}<0.99)$ vs. No VE 0.3 weeks; Fig. 2b, c). In contrast, all age matched, non-asbestos exposed control mice survived to the 70 week experimental endpoint (Fig. 2a, dashed lines).

\section{Asbestos exposure diminishes exercise participation}

We next assessed the time spent running per night and total distance run per night to determine whether 


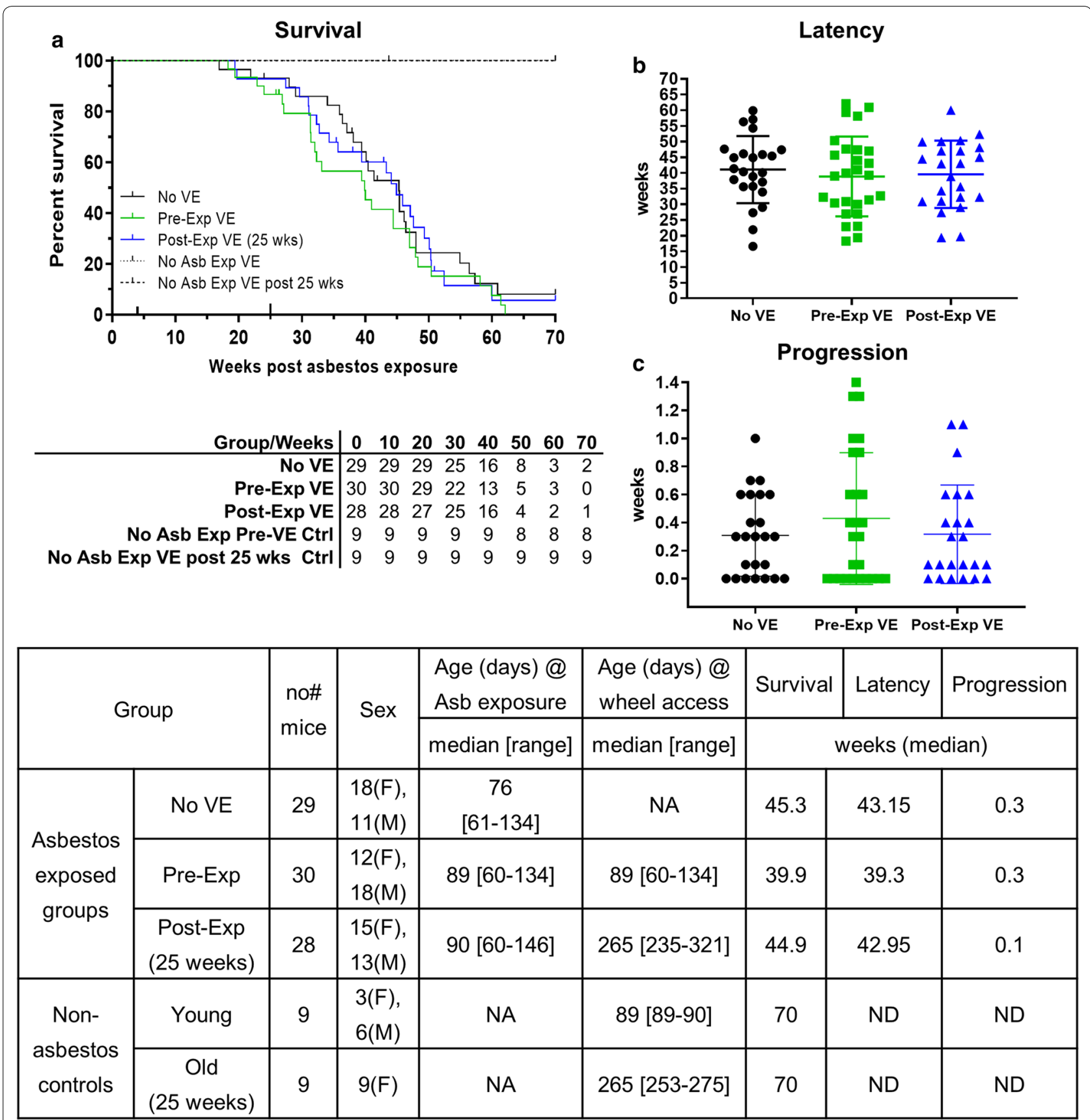

Fig. 2 Voluntary exercise does not affect asbestos related disease in MexTAg mice. Crocidolite asbestos (6 mg total) was injected into MexTAg mice via two intraperitoneal injections four weeks apart. a Kaplan-Meier plot depicting survival over a 70 week period with the number of mice at risk shown in the corresponding table. $\mathbf{b}$ ARD Latency (time from asbestos exposure to first signs of disease) and $\mathbf{c}$ progression (time from first signs of disease until cull) in asbestos-exposed groups. Table define experimental design and cohort characteristics. Data are censored for asbestos related deaths and show mean \pm SD. Log-rank (Mantel-Cox) analysis was used for survival. Kruskal-Wallis one-way ANOVA with Dunn's test for multiple comparisons for all other analyses. $p$ values $\leq 0.05$ were considered significant. A single age-matched, non-asbestos exposed mouse was culled at week 44 in an incident unrelated to VE

VE participation changed over time. All asbestos exposed mice displayed a progressive decline in exercise (time and distance run per night) over the 70-week experimental duration. For Pre-VE and Post-VE groups, a significant decline for both time spent running per night and total distance run per night, was observed 
after 15 weeks of access to VE (Pre-VE running time/ night: starting $16-20$ weeks, $\mathrm{p}=0.018$ and distance/ night: $\mathrm{p}=0.028$, sustained from 21 to 65 weeks, $\mathrm{p}<0.0001$; Post-VE time/night: starting 41-45 weeks $(\mathrm{p}=0.002)$ and distance/night $(\mathrm{p}=0.015)$, sustained from 46-70 weeks, both $\mathrm{p}<0.0001$; Fig. 3a-d). Whilst VE participation at 26-30 weeks was similar between Pre-VE and Post-VE groups, the Post-VE group showed a more profound and sustained reduction in exercise behaviours over time, with VE participation being almost negligible for mice surviving to 70 weeks (Fig. 3a, b vs. c, d).

To determine whether the decline in VE in asbestos exposed mice was affected by how old mice were when given access to VE, we repeated the experiment using non-asbestos exposed mice. Two groups, 'Young' or 'Old', were used as aged matched controls for Pre-VE (Young; 90 days at start of VE) and Post-VE (Old; 265 days at start of VE) groups respectively. Although a significant decrease in VE participation was observed within both control groups over time $(\mathrm{p}<0.01)$, this decrease was not as marked in comparison to their respective asbestos-exposed groups (1.51 to 4.28 -fold decrease vs 2.97 to 12.63 fold decrease non-asbestos vs asbestos exposed groups respectively). Magnitude of decrease in distance run per night was most pronounced in the Post-VE group (Fig. 3e-h).

This was further evident when we compared changes in VE over time between asbestos exposed VE groups and their respective control group, where both groups of asbestos exposed mice consistently spent significantly less time running, and ultimately travelled less distance per night, relative to their respective non-asbestos exposed control (Fig. 3i-n). Interestingly, VE participation in age matched, non-asbestos exposed controls in which VE was delayed (Old controls) was significantly higher $(\mathrm{p}<0.05)$ relative to the continuous VE (Young) control group (Fig. $3 \mathrm{~m}-\mathrm{n}$ ), suggesting that older mice might be more responsive to VE intervention. Taken together, these data indicate that asbestos exposed mice had reduced VE participation over time suggesting that the subclinical development of ARD after asbestos exposure had a greater impact on VE participation than age alone.

\section{Discussion}

Here we employed our asbestos induced MexTAg mouse model to assess the impact of VE on ARD following asbestos exposure. In contrast to other studies in which VE delayed tumour growth [13], the addition of VE (continuous or delayed) did not affect ARD development in our model. Mesothelioma is unusual in that the carcinogen, asbestos, induces a similar disease in mice and humans. This is rare in cancer research and presents an ideal opportunity to apply small animal models to advance mesothelioma prevention and treatment. However, model choice may explain why VE did not have any significant impact on ARD in asbestos exposed MexTAg mice. Previous studies demonstrating significant VE associated reduction in tumour incidence and growth employed mouse models employing intravenously or subcutaneous tumours [8-13]. In these studies, VE enhanced tumour suppression was associated with increased expression of p53 and mediators of apoptosis [9], or mobilisation and redistribution of NK cells in an epinephrine and IL-6 dependent manner [13]. In contrast, the MexTAg model involves induction of ARD in situ following asbestos exposure, where TAg expression phenocopies p16 loss, effectively bypassing p53/p16 mediated cell cycle control [21]. Therefore, the inherent genetic modifications that drive the oncogenic potential in transgenic models like MexTAg might mask any benefit induced by supportive adjuvant therapies like VE.

While we did not observe differences in ARD, we did observe significant differences in VE participation between, and within asbestos exposed and nonexposed groups. In particular, VE participation was higher in age matched, non-asbestos controls in which VE was delayed (Old) relative to asbestos exposed delayed VE and the continuous (Young) VE control group; indicating that asbestos exposure, rather than age, had a greater impact on the observed reduction in VE participation over time. We also observed a decrease in exercise prior to clinical signs of disease development in asbestos exposed mice. It is important to consider these data in context of the clinical setting, in which mesothelioma patients are often elderly, have a sedentary lifestyle and present with high disease

\footnotetext{
(See figure on next page.)

Fig. 3 Diminished capacity for VE after asbestos-exposure. MexTAg mice given access to VE either 2-weeks before (Pre-Exp VE), or 25-weeks after (Post-Exp VE) asbestos exposure displayed a significant and sustained decrease in both the amount of time spent running/night (a, $\mathbf{c})$ and total distance travelled/night (b, d) over a 70 week period. This was in contrast to age-matched, non-asbestos exposed MexTAg controls (e-h), which maintained significantly higher VE capacity over a similar time period. (i-n) Changes in VE over time between respective asbestos exposed and age-matched, non-asbestos exposed control groups (i, j: Pre-Exp VE vs Young Ctrl; $\mathbf{k}, \mathbf{l}$ : Post-Exp VE vs Old Ctrl and $\mathbf{m}, \mathbf{n}$ Young vs. Old controls). Table depicts fold-change over time. Data shown is mean \pm SD. (a-h) Data analysed using a non-parametric Kruskal-Wallis one way ANOVA with Dunn's test for multiple comparisons to start of VE. (i-n) Data analysed via non-parametric, mixed model ANOVA. ${ }^{*}=p<0.05,{ }^{* *}=p<0.01$, ${ }^{* * *}=p<0.001,{ }^{* * *}=p<0.0001$
} 


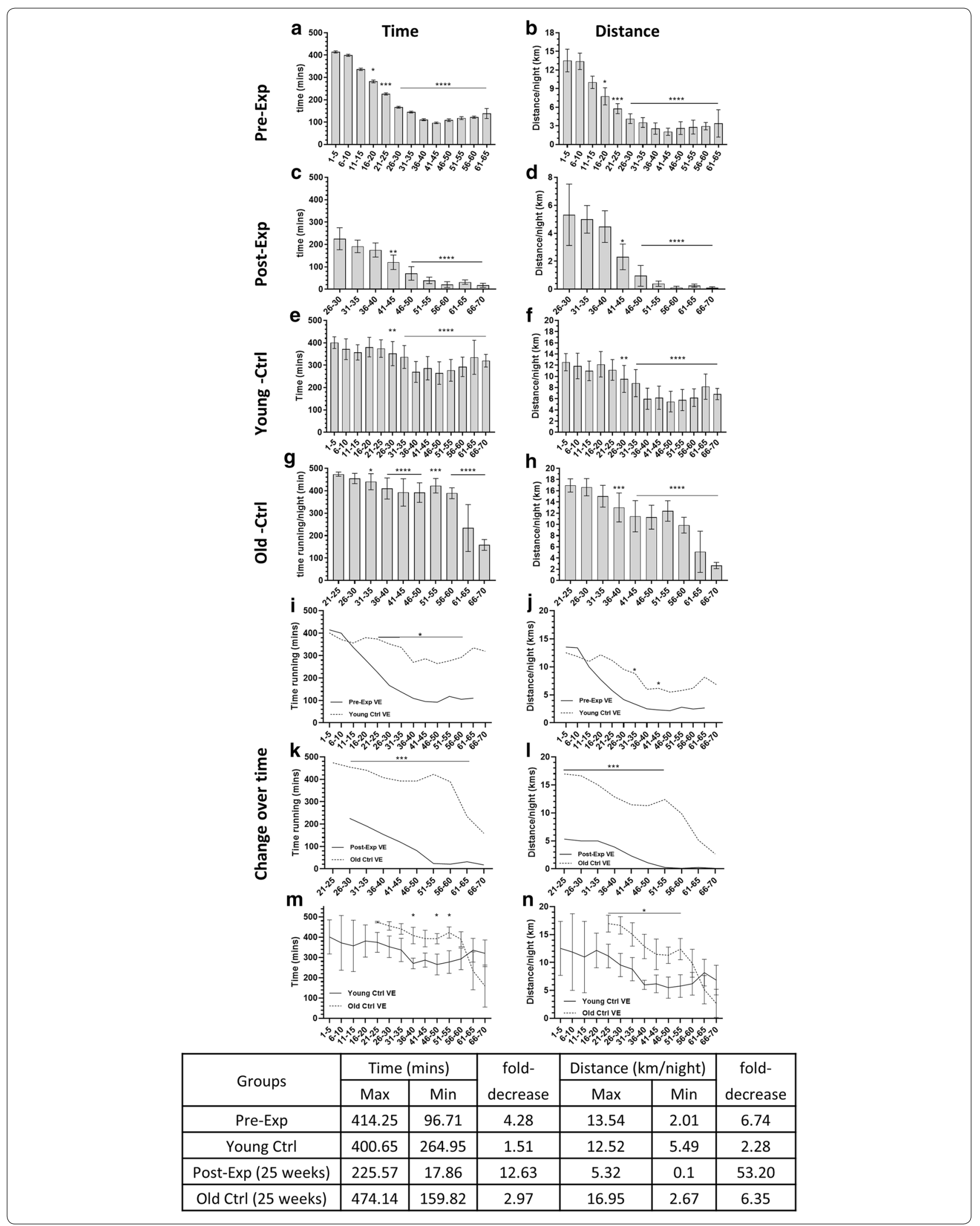


burden $[22,23]$. Together, these data suggest that the impact of VE on asbestos induced ARD might be better observed and modelled using aged mice. Alternatively, our recent human data [24] demonstrated that a short (six-week) tailored resistance exercise training program was well tolerated and beneficial to mesothelioma patients. As data in this study also indicated a decrease in exercise preceded disease detection, this may suggest that patients presenting with mesothelioma might benefit from additional support and rehabilitation at diagnosis.

In conclusion, the addition of continuous or delayed VE did not significantly affect ARD development in asbestos exposed MexTAg mice. Our data is in contrast to previous studies and highlights the importance of choosing an appropriate model and rigorously evaluating model parameters. Preclinical transplant models might be useful for 'proof of concept' studies, but as seen here, may not be applicable across different tumour types and may not phenocopy in situ development of human cancer. Additionally, our study highlights that exercise alone may not be sufficient to counteract the oncogenic potential of strong carcinogens like asbestos. As such, some pre-clinical studies may not accurately represent the clinical paradigm, particularly in the context of prevention style studies, and therefore have limited translational impact.

\section{Limitations}

Study-specific limitations include: 3 mice per cagetherefore, exercise data is not indicative of an individual mouse; Furthermore, similar ARD development across all groups might simply suggest that other modes of individualized exercise, rather than continuous VE used in this and other studies [8-13], might be more effective as adjunct supportive treatment for mesothelioma.

\section{Abbreviations \\ Asb: Crocidolite asbestos; FSD: First signs of disease; i.p: Intraperitoneal; MexTAg: Asbestos induced mesothelioma mouse model; $\mu \mathrm{m}$ : Micrometre; mg: Milligram; SD: Standard deviation; SV40: Simian Virus 40; TAg: Large T antigen from SV40; VE: Voluntary exercise; NHMRC: National Health and Medical Research Council. \\ Acknowledgements \\ The authors wish to acknowledge Wesley Wilson and Caitlin Tilsed (NCARD, Perth Western Australia) for the development of, and access to R scripts to perform mixed-model ANOVA analyses. We also thank Dr Shelly Gorman (Tel- ethon Kids Institute, Perth) for the loan of running wheels and thank Danika Hope, Sarah Dart and Tracy Seymour for assistance with animal monitoring.}

\section{Authors' contributions}

Conceptualization, SAF, CJPM and AKN; methodology, SAF, CJPM and KB; formal analysis, SAF, CJPM, KB, EM, RAL, RUN and AKN.; investigation, SAF, KB and EM; data curation, SAF, KB and CJPM.; writing — original draft preparation, SAF and CJPM; writing - review and editing, SAF, CJPM, KB, EM, RAL, RUN and
AKN; funding acquisition, AKN, SAF, RAL, EM, CJPM and RUN. All authors read and approved the final manuscript.

\section{Funding}

This research was funded in part from an NHMRC Centres of Research Excellence grant (APP1 107043), a Slater and Gordon Health Projects and Research Fund grant (AKN and SAF), charitable donations received by NCARD (Perth, Western Australia) and discretionary funding from Exercise Medicine Research Institute, Edith Cowan University. Perth, Australia. EM is a post-doctoral researcher supported by Kom Op Tegen Kanker (Stand Up to Cancer), the Flemish cancer society (Grant number: KotK_UA/2018/11519/1). We also acknowledge that EM was supported by the European Respiratory SocietyERS Short-Term Research Fellowship April 2019.

\section{Availability of data and materials}

The datasets used and/or analysed during the current study are available from the corresponding author on reasonable request.

\section{Ethics approval and consent to participate}

All animal experiments approved by The University of Western Australia Animal Ethics Committee (RA/3/100/1514), in accordance with the Australian National Health and Medical Research Council's (NHMRC) Code of Practice for the Use of Animals for Scientific Purposes.

\section{Consent for publication}

Not applicable.

\section{Competing interests}

The authors declare that they have no competing interests.

\section{Author details \\ ${ }^{1}$ National Centre for Asbestos Related Diseases (NCARD), Perth, Australia. ${ }^{2}$ School of Biomedical Sciences, University of Western Australia, Perth, Australia. ${ }^{3}$ Exercise Medicine Research Institute, Edith Cowan University, Perth, Australia. ${ }^{4}$ School of Medical and Health Sciences, Edith Cowan University, Joondalup, Australia. ${ }^{5}$ School of Human Movement and Nutrition Sciences, University of Queensland, St Lucia, QLD, Australia. ${ }^{6}$ Centre for Oncological Research, University of Antwerp, Antwerp, Belgium. ${ }^{7}$ School of Medicine, University of Western Australia, Perth, Australia.}

Received: 9 July 2020 Accepted: 10 September 2020

Published online: 15 September 2020

\section{References}

1. Zalcman G, Mazieres J, Margery J, Greillier L, Audigier-Valette C, MoroSibilot D, Molinier O, Corre R, Monnet I, Gounant V, et al. Bevacizumab for newly diagnosed pleural mesothelioma in the Mesothelioma Avastin Cisplatin Pemetrexed Study (MAPS): a randomised, controlled, open-label, phase 3 trial. Lancet. 2016;387(10026):1405-14.

2. Vogelzang NJ, Rusthoven JJ, Symanowski J, Denham C, Kaukel E, Ruffie P, Gatzemeier U, Boyer M, Emri S, Manegold C, et al. Phase III study of pemetrexed in combination with cisplatin versus cisplatin alone in patients with malignant pleural mesothelioma. J Clin Oncol. 2003;21 (14):2636-44.

3. Hansen J, de Klerk NH, Musk AW, Hobbs MS. Environmental exposure to crocidolite and mesothelioma: exposure-response relationships. Am J Respir Crit Care Med. 1998;157(1):69-75.

4. Rascoe PA, Jupiter D, Cao X, Littlejohn JE, Smythe WR. Molecular pathogenesis of malignant mesothelioma. Expert Rev Mol Med. 2012;14:e12.

5. Moore SC, Lee IM, Weiderpass E, Campbell PT, Sampson JN, Kitahara CM, Keadle SK, Arem H, de Gonzalez AB, Hartge P, et al. Association of leisuretime physical activity with risk of 26 types of cancer in 1.44 million adults. JAMA Intern Med. 2016;176(6):816-25.

6. Boyle T, Keegel T, Bull F, Heyworth J, Fritschi L. Physical activity and risks of proximal and distal colon cancers: a systematic review and meta-analysis. J Natl Cancer Inst. 2012;104(20):1548-61.

7. Lynch BM, Neilson HK, Friedenreich CM. Physical activity and breast cancer prevention. Recent Results Cancer Res. 2011;186:13-42.

8. Betof AS, Lascola CD, Weitzel D, Landon C, Scarbrough PM, Devi GR, Palmer G, Jones LW, Dewhirst MW: Modulation of murine breast tumor 
vascularity, hypoxia and chemotherapeutic response by exercise. J Natl Cancer Inst 2015. 107(5).

9. Higgins KA, Park D, Lee GY, Curran WJ, Deng X. Exercise-induced lung cancer regression: mechanistic findings from a mouse model. Cancer. 2014;120(21):3302-10

10. Jones LW, Antonelli J, Masko EM, Broadwater G, Lascola CD, Fels D, Dewhirst MW, Dyck JR, Nagendran J, Flores CT, et al. Exercise modulation of the host-tumor interaction in an orthotopic model of murine prostate cancer. J Appl Physiol. 2012;113(2):263-72.

11. McCullough DJ, Nguyen LM, Siemann DW, Behnke BJ. Effects of exercise training on tumor hypoxia and vascular function in the rodent preclinical orthotopic prostate cancer model. J Appl Physiol. 2013;115(12):1846-54.

12. McCullough DJ, Stabley JN, Siemann DW, Behnke BJ. Modulation of blood flow, hypoxia, and vascular function in orthotopic prostate tumors during exercise. J Natl Cancer Inst. 2014;106(4):036.

13. Pedersen L, Idorn M, Olofsson GH, Lauenborg B, Nookaew I, Hansen RH, Johannesen HH, Becker JC, Pedersen KS, Dethlefsen C, et al. Voluntary running suppresses tumor growth through epinephrine- and IL-6-dependent NK Cell mobilization and redistribution. Cell Metab. 2016;23(3):554-62.

14. Bay ML, Gehl J, Pedersen BK, Hojman P. Voluntary wheel running reduces the acute inflammatory response to liver carcinogen in a sex-specific manner. Cancer Prev Res. 2017;10(12):719-28.

15. Robinson C, van Bruggen I, Segal A, Dunham M, Sherwood A, Koentgen F, Robinson BW, Lake RA. A novel SV40 TAg transgenic model of asbestosinduced mesothelioma: malignant transformation is dose dependent. Cancer Res. 2006;66(22):10786-94.

16. Robinson C, Walsh A, Larma I, O'Halloran S, Nowak AK, Lake RA. MexTAg mice exposed to asbestos develop cancer that faithfully replicates key features of the pathogenesis of human mesothelioma. Eur I Cancer. 2011;47(1):151-61.
17. Australian code for the care and use of animals for scientific purposes In., 8th Ed edn. Canberra: National Health and Medical Research Council.; 2013.

18. Team RC. R: A language and environment for statistical computing. Austria: R Foundation for Statistical Computing. In. Vienna; 2018.

19. Henrik Singmann BB, Jake Westfall and Frederik Aust.: afex: Analysis of Factorial Experiments. R package version 0.25-1. edn In. In.; 2019.

20. Lenth R: emmeans: Estimated Marginal Means, aka Least-Squares Means. In., R package version 1.4.3.01 edn.. In.; 2019.

21. Robinson C, Dick IM, Wise MJ, Holloway A, Diyagama D, Robinson BW, Creaney J, Lake RA. Consistent gene expression profiles in MexTAg transgenic mouse and wild type mouse asbestos-induced mesothelioma. BMC Cancer. 2015;15:983.

22. Jeffery E, Lee YCG, Newton RU, Lyons-Wall P, McVeigh J, Nowak AK, Cheah HM, Nguyen B, Fitzgerald DB, Creaney J, et al. Body composition and nutritional status in malignant pleural mesothelioma: implications for activity levels and quality of life. Eur J Clin Nutr. 2019;73(10):1412-21.

23. Jeffery E, Lee YG, McVeigh J, Straker L, Wooding T, Newton RU, PeddleMcIntyre C. Feasibility of objectively measured physical activity and sedentary behavior in patients with malignant pleural effusion. Support Care Cancer. 2017;25(10):3133-41.

24. Peddle-McIntyre CJ, Je LY, Nowak AK, Nguyen B, Newton RU: Six-week exercise intervention in patients with malignant pleural disease improves physical function, muscular strength and appendicular lean mass. In: Thoracic Society of Australia New Zealand. Adelaide; 2018.

\section{Publisher's Note}

Springer Nature remains neutral with regard to jurisdictional claims in published maps and institutional affiliations.
Ready to submit your research? Choose BMC and benefit from:

- fast, convenient online submission

- thorough peer review by experienced researchers in your field

- rapid publication on acceptance

- support for research data, including large and complex data types

- gold Open Access which fosters wider collaboration and increased citations

- maximum visibility for your research: over $100 \mathrm{M}$ website views per year

At BMC, research is always in progress.

Learn more biomedcentral.com/submissions 